\title{
Three-dimensional simulations of compressible tearing instability
}

\author{
Simone Landi \\ Dipartimento di Astronomia a Scienza dello Spazio, Università degli Studi di Firenze, Largo E. Fermi 2, \\ I-50125 Firenze, Italy \\ Pasquale Londrillo \\ Istituto Nazionale di AstroFisica (INAF) - Osservatorio Astronomico di Bologna, Via Ranzani 1, I-40127 \\ Bologna, Italy \\ Marco Velli \\ Jet Propulsion Laboratory (JPL), California Institute of Technology, 4800 Oak Grove Drive, Pasadena, \\ California 91109, USA \\ Lapo Bettarini \\ Dipartimento di Astronomia a Scienza dello Spazio, Università degli Studi di Firenze, Largo E. Fermi 2, \\ I-50125 Firenze, Italy
}

(Received 21 September 2007; accepted 9 November 2007; published online 2 January 2008)

\begin{abstract}
Three-dimensional numerical simulations of the tearing instability in the framework of compressible and resistive magnetohydrodynamics are presented. Simulations have been performed with a novel Eulerian conservative high order code, including an explicit resistivity, which uses implicit high order numerical schemes having higher spectral resolution than classical schemes. The linear and non linear evolution of the tearing instability has been followed for force-free and pressure-balanced initial equilibrium configurations. Pressure equilibrium configurations are subject to a secondary instability which drives the system toward a quasi two dimensional structure oriented perpendicularly to the initial configuration. The development of secondary instabilities is suppressed by a guide field allowing the coalescence instability to fully develop in the system. Force-free initial configurations follow an intermediate path with respect the previous cases: Strong coalescence of magnetic islands, due to the non linear evolution of the tearing instability, is observed before the system enters in a phase dominated by $3 \mathrm{D}$ modes. The histories of the differing initial current-sheet equilibria have counterparts in the energy spectra that, for all three cases, are observed to be strongly anisotropic. (C) 2008 American Institute of Physics. [DOI: 10.1063/1.2825006]
\end{abstract}

\section{INTRODUCTION}

It is widely accepted that magnetic reconnection plays an important role in both astrophysical and laboratory plasmas. During the reconnection process, energy stored in the magnetic field is converted into heat and flow energy in a short time. In this sense, it is a good candidate to explain explosive events, such as solar flares and coronal mass ejections, as well as less dramatic phenomena like solar corona heating. ${ }^{1,2}$ Plasma instabilities associated with finite resistivity also have a strong influence on the stability fusion devices in the reverse field pinch configuration. ${ }^{3}$

The basic instability of the current sheet is the tearing instability. The linear properties were first studied analytically by Furth et $a l .{ }^{4}$ in the early 1960 s, and numerically by Wesson $^{5}$ and Steinolfson and van Hoven. ${ }^{6}$ The nonlinear evolution of resistive instabilities produces turbulence by energy transfer toward higher wave numbers as well as an inverse cascade via coalescence processes that produces magnetic islands with larger and larger size. Coalescence has been extensively studied in incompressible magnetohydrodynamics (MHD) starting from two-dimensional configurations in which the magnetic islands are produced by the superposition of current filaments embedded in a current sheet producing antiparallel magnetic fields. The overall current distribution in this case has a maximum in correspondence of the $\mathrm{O}$-points and a minimum at the $\mathrm{X}$-point, and coalescence is driven by the attraction of parallel current filaments. ${ }^{7-11}$ The tearing instability, on the other hand, leads to the formation of magnetic islands where current maxima are located at $\mathrm{X}$-points and minima at O-points. In such configurations, coalescence is driven by the stretching of the most intense X-points. ${ }^{12-14}$

When moving from two to three dimensions, it is found that many hydrodynamic and MHD configurations may become unstable to the so called secondary instability. ${ }^{15-21}$ The initial equilibrium starts off being unstable to a twodimensional (2D) instability (primary instability) that grows, driving the system to a new $2 \mathrm{D}$ configuration that is itself unstable to ideal three-dimensional (3D) modes that can drive the system toward a turbulent state. It has been shown that the 2D nonlinearly saturated configuration of an initial current sheet subjected to the tearing instability develops an ideal instability that has growth rates exceeding the reconnection rate. ${ }^{16,18}$ A 3D secondary instability of ideal nature has also been observed in a plane current-vortex sheet, ${ }^{17,22,23}$ a configuration in which a vortex sheet is superposed on a current sheet that may be unstable to both ideal and resistive primary modes. ${ }^{24-26}$

The presence of a constant magnetic field parallel to the current density, a guide field, can affect the growth of the secondary instability, as conjectured by Dahlburg and Einaudi. ${ }^{18}$ 3D incompressible MHD simulations of the tear- 
ing instability have shown that the presence of a guide field makes the evolution more similar to that observed in the $2 \mathrm{D}$ configuration. ${ }^{27}$ The simulations show a $2 \mathrm{D}$ behavior at large scales (formation of magnetic island with increasing size) and 3D structuring with current filamentation at smaller scale. More recently, Dahlburg et al. ${ }^{28}$ studied the linear and nonlinear evolution of a current sheet where a guide field is present. They found that for a wide interval of values of the guide field intensity, the initial current sheet configuration is still subjected to the secondary instability.

The scope of this paper is to extend the study of the linear and nonlinear tearing instabilities to a compressible plasma. In particular, we are interested in exploring under which conditions a secondary instability occurs. For this reason, we start from the same basic initial configuration used in the previous works quoted above, i.e., a current sheet where magnetic forces are counterbalanced by a plasma pressure gradient. At the same time, we are interested in looking at other possible initial configurations where a current sheet, possibly unstable to tearing, is present. In particular, here we consider a force-free equilibrium where the current sheet is formed by a rotation of the cross-sheet component of the magnetic field. In this case, a nonvanishing component of the magnetic field perpendicular to the plane formed by the asymptotic magnetic field and the cross-sheet direction is present only inside the current sheet. In this sense, the forcefree equilibrium is a configuration that is in the middle between the pressure equilibrium configurations where a guide field is present or not. A force-free configuration where the magnetic field does not vanish across the current sheet can have interesting astrophysical applications, for example the heliospheric current sheet. ${ }^{29}$

In compressible MHD, the dynamical activity following the current sheet instability leads to the formation of nonlinear waves that can steepen into discontinuous modes. Shockcapturing numerical schemes, where numerical diffusivity is properly designed to be effective only at points of discontinuity, leaving unaffected smooth flow regions, are then appropriate. At the same time, the presence of turbulent small scales activity and of kinetic terms as well, such as resistivity and Hall effect, requires high-order numerical approximations to assure sufficient space resolution and vanishing numerical diffusivity. To that purpose, compact (or implicit) differentiation schemes ${ }^{30}$ having spectral-like resolution provide significant advantages over the corresponding explicit finite-difference schemes. In the fluid dynamic case, much work has been devoted to reconcile shock-capturing properties with high-order, spectral-like differentiation schemes, but in the compressible MHD case, numerical achievements are still scarce in this research area. For that purpose, we have designed specific upwinding procedures and compact differentiation schemes that turn out to be adequate in the present study of current sheet dynamical evolution.

The paper is organized as follows. Section II presents and discusses the code used for the numerical simulations. Since most computational tools and technical aspects are documented elsewhere, only the main procedures and the relevant properties of the code are presented. A specific documentation on the spectral properties of the differentia- tion schemes is reported in the Appendix. In Sec. III, we describe normalizations, the initial equilibrium configurations, and perturbations in the numerical simulations. The linear analysis of the tearing instability is discussed in Sec. IV, while the 2D nonlinear evolution, used here as a benchmark for the 3D dynamics, is presented in Sec. V. 3D simulations are presented in Sec. VI, and a discussion of the results and conclusions are presented in Sec. VII.

\section{THE NUMERICAL SCHEME}

To solve the compressible MHD equations in conservative form, we have designed an Eulerian conservative high order (ECHO) code, based on the upwind constrained transport (UCT) general methodology proposed in Refs. 31 and 32. A related version of the code has been designed for special relativistic ${ }^{33}$ and general relativistic ideal MHD equations. ${ }^{34}$ With respect to previous versions, in the code an explicit plasma resistivity has been introduced and space resolution has been improved by using high-order compact (or implicit) difference schemes ${ }^{30}$ to approximate flux derivatives. Here we briefly summarize the main procedures adopted in the code by focusing on the specific aspects related to the present physical application.

In a conservative form, the set of the MHD equations can be represented by two coupled subsystems, the first one containing space operators in divergence form, and the second one, specific to the magnetic field evolution, containing space operators in the curl form,

$$
\begin{aligned}
& \frac{\partial \mathbf{U}}{\partial t}+\nabla \cdot \mathbf{F}(\mathbf{w})=0, \\
& \frac{\partial \mathbf{B}}{\partial t}+\nabla \times \mathbf{E}(\mathbf{w})=0,
\end{aligned}
$$

constrained by the nonevolutionary condition for the magnetic field $\nabla \cdot \mathbf{B}=0$. In the set of Eqs. (1) and (2), $\mathbf{U}$ is the five-component array of conservative variables $\mathbf{U}=[\rho, \mathbf{q}, e]^{T}$ with $\rho$ the density, $\mathbf{q}=\rho \mathbf{v}$ the three components of the momentum, $\mathbf{v}$ the fluid velocity, and $e=p /(\gamma-1)+\rho v^{2} / 2$ $+B^{2} / 2$ the total energy for a perfect gas equation state, with $p$ its pressure and $\gamma$ the adiabatic index. In the upwind literature, the nonconservative variables $\mathbf{P}=[\rho, \mathbf{v}, p]^{T}$ are usually referred to as primitives, the magnetic field components acting both as conservative or primitive variables. The corresponding flux components are given by $\mathbf{F}=\left[\mathbf{q}, M_{i j}, \mathbf{H}\right]^{T}$ with

$$
\begin{aligned}
& M_{i j}=v_{i} q_{j}+\Pi \delta_{i j}-B_{i} B_{j}, \\
& \mathbf{H}=\mathbf{v}(e+\Pi)-(\mathbf{v} \cdot \mathbf{B}) \mathbf{B}-\frac{c}{\sigma} \mathbf{B} \times \mathbf{J},
\end{aligned}
$$

where in Eq. (4) we have introduced the Joule dissipation of the current density $\mathbf{J}=c / 4 \pi(\nabla \times \mathbf{B})$ due to a finite conductivity $\sigma . \Pi=p+B^{2} / 2$ is the total (fluid plus magnetic) pressure. In Eq. (2), the induction equation for the magnetic field, the corresponding flux is simply the electric field, 


$$
\mathbf{E}=-\mathbf{v} \times \mathbf{B}+\frac{c}{\sigma} \mathbf{J}=-\mathbf{v} \times \mathbf{B}+\eta \nabla \times \mathbf{B},
$$

where $\eta=c^{2} /(4 \pi \sigma)$ is the magnetic diffusivity.

Upwind schemes designed for fluid dynamics can be extended to the MHD system by application of the following numerical procedures: ${ }^{31}$

1. To compute flux derivatives along each coordinate direction, we denote generically by $u_{i}$ any scalar component of the $(\mathbf{U}, \mathbf{B})$ state variable discretized on the generic $x_{i}$ coordinate axis with grid size $\Delta x$, and by $f_{i}=f\left(u_{i}\right)$ a corresponding flux component.

2. In the first set Eq. (1) of MHD equations, like in the Euler equations of fluid dynamics, each conservative (or primitive) variable, $u_{i}$, is first reconstructed (REC) at the corresponding cell interface $x_{i+1 / 2}$ using left-biased (L) or right-biased $(\mathrm{R})$ interpolation. In symbolic form,

$u_{i} \rightarrow\left[u^{L}, u^{R}\right]_{i+1 / 2}$.

Correspondingly, each flux component $f(u)$ turns out to be a two-sided interpolated function,

$f_{i}=f\left(u_{i}\right) \rightarrow\left[f^{L}, f^{R}\right]_{i+1 / 2}$.

To achieve shock-capturing properties, one-sided interpolation schemes are required to have specific nonoscillatory properties, in order to detect flow discontinuities and to represent them in a monotonic way. To that end, a large body of numerical work, mainly for fluiddynamical systems, is available. In the ECHO code, besides the weighted essentially non-oscillatory (WENO) ${ }^{35}$ explicit reconstruction schemes, we have implemented an upwind compact REC interpolation, with a proper limiter to enforce monotonicity and to avoid Gibbs oscillations near a discontinuity. ${ }^{36}$ Spectral properties of the REC procedure are presented in the Appendix.

3. After the reconstruction phase, in a second main step, the two-valued function $\left[f^{L}, f^{R}\right]$ is combined into a onevalued, continuous upwind flux via an approximate Riemann solver. Here we adopt the simplest Lax-Friedrichs formula, giving

$f_{\mathrm{LF}}\left(u^{L}, u^{R}\right)=\frac{1}{2}\left[f^{L}+f^{R}-\alpha_{x}\left(u^{R}-u^{L}\right)\right]$,

where $\alpha_{x}=\left|v_{x}\right|+\lambda_{x}$ and $\lambda_{x}$ is the local average of the maximum eigenvalue (the characteristic wave speed) of the flux Jacobian. In the MHD case, $\lambda_{x}=\left(V_{f}\right)_{x}$, where $V_{f}$ is the fast magnetosonic speed.

4. We notice that, if $r$ denotes the formal order of the interpolating approximations, the $\left(u^{R}-u^{L}\right)$ term in flux formulas gives a diffusive or dispersive contribution of size $O(\Delta x)^{r}$ in smooth regions, reducing to first order $O(\Delta x)$ at a flow discontinuity. In most of the present simulations, we adopt REC interpolation of $r=7$ order.

5 . $r$-order interpolation of $u$ variables provides a corresponding high-order approximation for the flux function at the relevant cell interface,

$f_{i+1 / 2}=f\left(x_{i+1 / 2}\right)+O(\Delta x)^{r}$,

but the flux difference, $\frac{f_{i+1 / 2}-f_{i-1 / 2}}{\Delta x}=f_{i}^{\prime}+O(\Delta x)^{2}$,

gives only a second order approximation of the flux derivative $f_{i}^{\prime}$, irrespective of the chosen interpolation accuracy. To achieve high-order approximations even for flux derivatives, a second main reconstruction procedure (DER) is required, by combining flux values at intercell points to recover a numerical derivative function $f_{i+1 / 2}$ $\rightarrow \hat{f}_{i+1 / 2}$ having the desired property,

$\frac{\hat{f}_{i+1 / 2}-\hat{f}_{i-1 / 2}}{\Delta x}=f_{i}^{\prime}+O(\Delta x)^{k}$.

In flows not dominated by strong discontinuities, as in the present application, the DER reconstruction step can be implemented by using centered compact finite differences of $k=r+1$ order, ${ }^{30}$ and no limiter is now required. The spectral properties of the compact DER procedure are presented in the Appendix.

6. The same procedures apply to the induction equation, where a proper staggered discretization and a specific 2D Riemann solver are needed to take into account the specific curl form of the differential operators and of the related conservation laws based on the Stokes theorem. ${ }^{32}$ We notice that the above upwind flux reconstruction applies only to the ideal set of MHD equations. The components of the current vector $\mathbf{J}$ entering in the Ohm law of the induction equation and in the corresponding Joule dissipation term of the energy equation have been approximated by a simple fourth order central finite-difference scheme. This numerical representation of the resistivity turns out to be effective and consistent with the overall scheme as long as the resistivity coefficient $(i)$ is sufficiently larger than the $O(\Delta x)^{r}$ numerical diffusivity and at a time (ii) sufficiently small to be effective only at the smallest resolved scales.

7. Finally, time integration of the overall set of MHD equations is performed by a third order Runge-Kutta scheme, ${ }^{37}$ under the usual Courant-Friedrichs-Lewy (CFL) stability condition (here in the most restrictive form),

$\Delta t<\min \left[\frac{\Delta}{\alpha}, \frac{\Delta^{2}}{\eta}\right]$,

where $\Delta$ is a relevant grid size and $\alpha$ denotes the maximum of the $\alpha_{j}$ eigenvalues among the $j=x, y, z$ coordinate directions.

The ECHO integration scheme, as summarized above, by construction shares the following main properties:

1. Global conservation laws are numerically satisfied.

2. The local divergence-free condition, if satisfied in the initial condition, is preserved identically in time to within machine accuracy.

3. Small-scale flow structures are well resolved.

4. The resistivity terms, even with normalized coefficients $\eta \ll \Delta$, can be fully appreciated against numerical diffusivity. 


\section{SETUP OF THE SIMULATIONS}

We consider two kinds of equilibrium configurations as starting points for the tearing instability. The first is a forcefree (FF) equilibrium where the magnetic field inside the current sheet rotates, the second configuration has a constant total pressure, i.e., the magnetic pressure is counterbalanced by a pressure gradient (pressure equilibrium or PE). The PE configuration also allows the presence of a uniform guide field in the direction normal to the current sheet. In the following, physical quantities are rendered dimensionless, using a characteristic length scale $L_{0}$, a characteristic density $\rho_{0}$, and a characteristic magnetic field strength $B_{0}$. Velocities are thus expressed in terms of the Alfvén speed $a_{0}=B_{0} / \sqrt{4 \pi \rho_{0}}$ and time is measured in term of $t_{0}=L_{0} / a_{0}$. Fluid pressure is expressed in term of the plasma $\beta=8 \pi P_{0} / B_{0}^{2}$ and temperature is thus expressed in term of the sound speed $\gamma T_{0}=c_{s}^{2}$ with $c_{s}^{2} / a_{0}^{2}=\gamma \beta / 2$. The magnetic diffusivity is expressed in terms of the Lundquist number $R_{\eta}=a_{0} L / \eta$.

\section{A. Force-free equilibrium}

We consider a plasma at rest permeated by a background magnetic field sheared along the $y$ direction with the current sheet in the middle of the simulation domain. Far from the current sheet, the magnetic field is directed along the $x$ direction and is given by

$$
B_{0 x}=\tanh (\delta y) \text {. }
$$

The parameter $\delta$ represents the inverse of the thickness of the current sheet. The equilibrium is ensured by a magnetic field directed along the $z$ direction such that

$$
\nabla\left(B_{0 z}^{2}+B_{0 x}^{2}\right)=0,
$$

i.e.,

$$
B_{0 z}=\frac{1}{\cosh (\delta y)} .
$$

The plasma density is assumed uniform and equal to 1 , while the plasma pressure is $p=0.5 \beta$, and the sound speed is simply $c_{s}^{2}=0.5 \gamma \beta$ ( $\gamma$, the adiabatic index, is here taken to be $\gamma$ $=5 / 3$ ). This configuration is an equilibrium configuration in the limit of zero resistivity, $1 / R_{\eta}=0$.

\section{B. Pressure equilibrium}

The equilibrium can also be ensured by a gas pressure gradient with

$$
p+B_{x}^{2} / 2=\text { const. }
$$

The constant is chosen to have a given plasma $\beta$ far from the current sheet. In practice, we choose

$$
p=\frac{1}{2}\left(\beta+1-B_{x}^{2}\right) .
$$

In this configuration, a uniform guide field directed along the $z$ direction, $B_{z 0}$, may be added.

In the $x$ and $z$ direction we assume periodic boundary conditions, while for the $y$ direction we have assumed a reflecting boundary using a ghost-cell technique with the appropriate symmetries for the basic fields.

\section{Perturbation}

The equilibrium is perturbed with a velocity fluctuation in the $y$ direction of amplitude $\epsilon$ and wave vectors $k_{x}$ and $k_{z}$ along the periodic directions $x$ and $z$. Along the $y$ direction, the perturbation vanishes far from the current sheet and is odd across the magnetic field shear as required by the classical tearing mode. The functional form of such perturbation is the following:

$$
v_{1 y}=\sum_{k_{x}=k_{x m}}^{k_{x M}} \sum_{k_{z}=-k_{z M}}^{k_{z M}} \epsilon \frac{\tanh (\delta y)}{\cosh (\delta y)} \sin \left(k_{x} x+k_{z} z+\phi_{k_{x}, k_{z}}\right),
$$

with $\phi_{k_{x}, k_{z}}$ a random phase for each mode, $k_{x m}$ and $k_{x M}$ the minimum and maximum values for the $x$ component of the wave vectors, and $k_{z M}$ the maximum for the $z$ component. The compressible, resistive MHD equations are solved in a domain $\left[0, L_{x}\right] \times\left[-L_{y}, L_{y}\right] \times\left[0, L_{z}\right]$ with resolution $N_{x}, N_{y}$, and $N_{z}$, respectively. The wave vectors can be expressed in terms of the discretized wave numbers,

$$
k_{x}=\frac{2 \pi}{L_{x}} m, \quad k_{z}=\frac{2 \pi}{L_{z}} n .
$$

\section{Parameters and adopted resolution}

If not differently specified, for the simulations described in the subsequent sections, we have assumed a Lundquist number $R_{\eta}=5000$ and a current sheet thickness $\delta=10$. We have considered the following initial configurations: (i) A pressure equilibrium with the presence of a guide field whose intensity is $B_{z 0}=1$, (ii) a pressure equilibrium without guide field, and (iii) a force-free configuration. For all the three basic configurations, we have considered both a high and low plasma beta with $\beta=5.0$ and 0.2 , respectively.

For the 2D linear and nonlinear analysis, we have adopted a simulation box of dimensions $L_{x}=6 \pi$ and $L_{y}$ $=\pi / 2$ with resolutions $N_{x}=128$ and $N_{y}=256$, respectively. For the 3D simulations, we have set $L_{z}=2 \pi$ and $N_{z}=64$. The values of $L_{y}$ and $N_{y}$ have been chosen taking into account that the boundaries along the $y$ direction must be positioned sufficiently far from the current sheet in order to prevent stabilizing effects from the walls. At the same time, the boundaries should not be so far away as to require a huge number of grid points, in order to resolve the field gradients across the current sheet. The values of $L_{y}$ and $N_{y}$ adopted give a good compromise between position of the boundary, resolution of the field gradients, and CPU time expense. Since the goal of the simulations is to follow, in the nonlinear regime, both the direct cascade toward higher wave vectors and the coalescence process toward larger scales, the length of the simulation box along the current sheet has been chosen large enough to fit the mode with the maximum linear growth rate. From the linear analysis, we will see that, for the aforementioned values of the parameters $\delta$ and $R_{\eta}$, the maximum growth rate occurs for wave vectors $k_{x}$ in the range $[1.5,3]$ (see Fig. 1). With a box of dimension $L_{x}=6 \pi$, such wave vectors correspond approximately to wave numbers $m$ in the range [4-9] of the fundamental one $k_{x 0}=1 / 3$. 

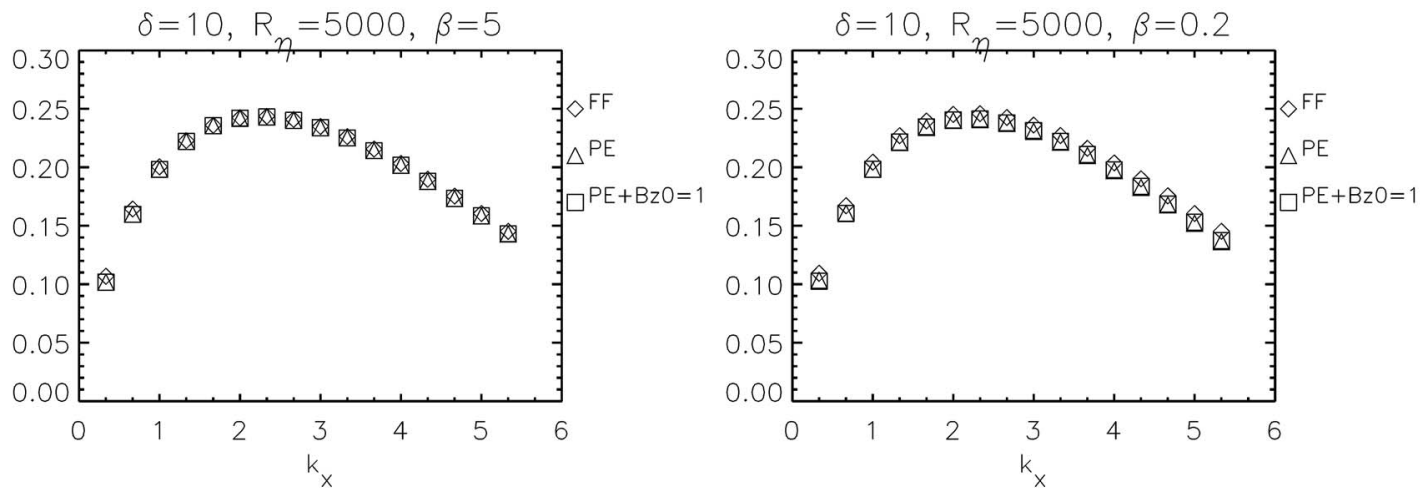

FIG. 1. Growth rates of the most unstable modes as a function of the wave vectors $k_{x}$ obtained by the linear code for the force-free (FF) configuration and for the pressure equilibrium (PE) configuration also in the presence of a guide field $B_{z 0}=1(\mathrm{PE}+\mathrm{Bz} 0=1)$. Results are reported for $\beta=5.0$ (left panel) and for $\beta=0.2$ (right panel).

\section{LINEAR ANALYSIS}

Insight into what happens in the first stages of evolution can be obtained by considering small perturbations $f_{1}$ for the main fields $\rho, T, \mathbf{B}$, and $\mathbf{v}$ and linearizing,

$$
\begin{aligned}
\frac{\partial \rho_{1}}{\partial t}= & -\nabla \cdot\left(\rho_{1} \mathbf{v}_{\mathbf{0}}\right)-\nabla \cdot\left(\rho_{0} \mathbf{v}_{\mathbf{1}}\right), \\
\frac{\partial T_{1}}{\partial t}= & -\mathbf{v}_{\mathbf{0}} \cdot \nabla T_{1}-\mathbf{v}_{\mathbf{1}} \cdot \nabla T_{0}-(\gamma-1) \\
& \times\left(T_{1} \nabla \cdot \mathbf{v}_{\mathbf{0}}+T_{0} \nabla \cdot \mathbf{v}_{\mathbf{1}}\right),
\end{aligned}
$$

$$
\begin{aligned}
\frac{\partial \mathbf{v}_{\mathbf{1}}}{\partial t}= & -\frac{1}{\rho_{0}} \nabla\left(\rho_{0} T_{1}+\rho_{1} T_{0}\right)+\left(\nabla \times \mathbf{B}_{\mathbf{0}}\right) \times \mathbf{B}_{\mathbf{1}} \\
& +\left(\nabla \times \mathbf{B}_{\mathbf{1}}\right) \times \mathbf{B}_{\mathbf{0}},
\end{aligned}
$$

$$
\frac{\partial \mathbf{B}_{\mathbf{1}}}{\partial t}=\nabla \times\left(\mathbf{v}_{\mathbf{1}} \times \mathbf{B}_{\mathbf{0}}\right)+\nabla \times\left(\mathbf{v}_{\mathbf{0}} \times \mathbf{B}_{\mathbf{1}}\right)+\frac{1}{R_{\eta}} \nabla^{2} \mathbf{B}_{\mathbf{1}} .
$$

These equations have solutions in the form

$$
\begin{aligned}
& \mathbf{B}_{1}(x, y, t)=\hat{B}_{1}(y) \mathrm{e}^{j k_{x} x} \mathrm{e}^{\Gamma t}, \\
& \mathbf{v}_{1}(x, y, t)=\hat{v}_{1}(y) \mathrm{e}^{j k_{x} x} \mathrm{e}^{\Gamma t},
\end{aligned}
$$

with $\Gamma$ the growth rate and $\hat{\mathbf{v}}_{1}, \hat{\mathbf{B}}_{1}$ the spatial dependence of the corresponding eigenmodes.

The set of the linearized equations is solved numerically. The numerical method here employed is the same used, for example, in Landi et al. ${ }^{38}$ for the set of fully nonlinear compressible MHD equations. The equations are integrated in a rectangular box of dimensions $L_{x} \times L_{y}$ assuming $z$ as an ignorable coordinate. In the periodic direction $x$, spatial integration is performed by spectral methods, while in the $y$ direction spatial integration is performed by using a sixth order cell centered compact difference scheme ${ }^{30}$ for the first and second derivatives. The time integration is performed by a third order Runge-Kutta method. The divergenceless condition for the magnetic field is ensured by integrating the $z$ component of the vector potential $\phi_{1}(x, y)$ instead of the $x$ and $y$ component of the perturbed magnetic field $B_{1 x}$ and $B_{1 y}$. The equation for $\phi_{1}$ is

$$
\frac{\partial \phi_{1}}{\partial t}=\left(\mathbf{v}_{\mathbf{1}} \times \mathbf{B}_{\mathbf{0}}\right)_{z}+\left(\mathbf{v}_{\mathbf{0}} \times \mathbf{B}_{\mathbf{1}}\right)_{z},
$$

with

$$
\begin{aligned}
& B_{1 x}=\frac{\partial \phi_{1}}{\partial y}, \\
& B_{1 y}=-\frac{\partial \phi_{1}}{\partial x} .
\end{aligned}
$$

With the linear code, we compute the growth rates for the most unstable modes, considering the equilibrium configurations described in Secs. III A and III B with the parameters given in Sec. III D, while for the initial perturbations we used Eq. (18) with $k_{z}=0$ and the wave number $m$ in the interval $1-16$, corresponding to wave vectors in the range $k_{x} \in[0.3-5.3]$. The obtained dispersion relations for the high and low plasma beta regimes (reported in the left and right panels of Fig. 1, respectively) do not seem to be affected by the nature of the background equilibrium configuration nor by the plasma pressure. The maximum growth rate for all the simulations is about 0.25 for wave numbers in the interval 6-9 corresponding to wave vectors in the range $2-3$.

\section{TWO-DIMENSIONAL NONLINEAR SIMULATIONS}

Before we start discussing the more interesting threedimensional case, it is important to understand the linear and nonlinear evolution of the aforementioned equilibria in 2D, i.e., assuming that the system is invariant along the $z$ direction. The exercise is useful in the sense that, on the one hand, we can verify the robustness of the numerical schemes 


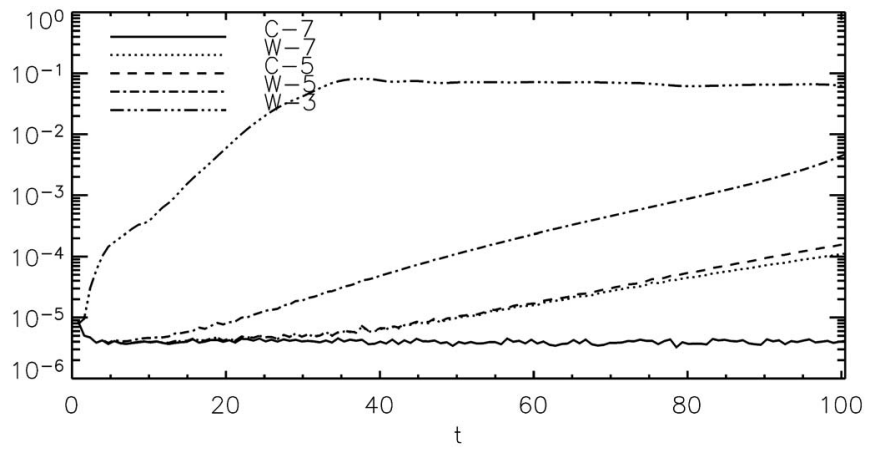

FIG. 2. Numerical dissipation for various schemes as measured by the global fluctuating energy as a function of time for the force-free configuration in a system where explicit magnetic diffusivity has been set equal to zero. The schemes used were the following: Seventh and fifth order compact schemes (C-7 and C-5, respectively), seventh, fifth, and third order explicit WENO schemes (W-7, W-5, and W-3, respectively).

adopted in describing the evolution of the equilibria, and, on the other hand, the nonlinear stage of the two-dimensional evolution can be used as reference for the more complex three-dimensional dynamics.

As seen in Sec. II, the code used here is able to handle different REC and DER procedures. The most appropriate scheme for the problem must take into account the implicit numerical dissipation as well as the spectral properties of the numerical scheme. As a first test, we have performed a set of simulations of the basic configurations without adding any explicit diffusivity (i.e., setting $R_{\eta}=\infty$ ). We have initialized the system with a small dose of perturbations with $\epsilon=10^{-4}$ in the wave-number range $m \in[1,12]$. Results using different schemes are shown in Fig. 2, where we report the amplitude of the fluctuating magnetic energy as a function of time. From the simulations, we note that except for the seventh order compact scheme (C-7), numerical dissipation leads to an almost exponential increase of the fluctuating energy. The corresponding growth rate is $\Gamma \simeq 0.04$ for the fifth order compact scheme (C-5) and for the seventh order explicit WENO scheme $(\mathrm{W}-7), \Gamma \simeq 0.08$ for the fifth order compact scheme (W5), and $\Gamma \simeq 0.27$ for the third order WENO scheme (W-3).

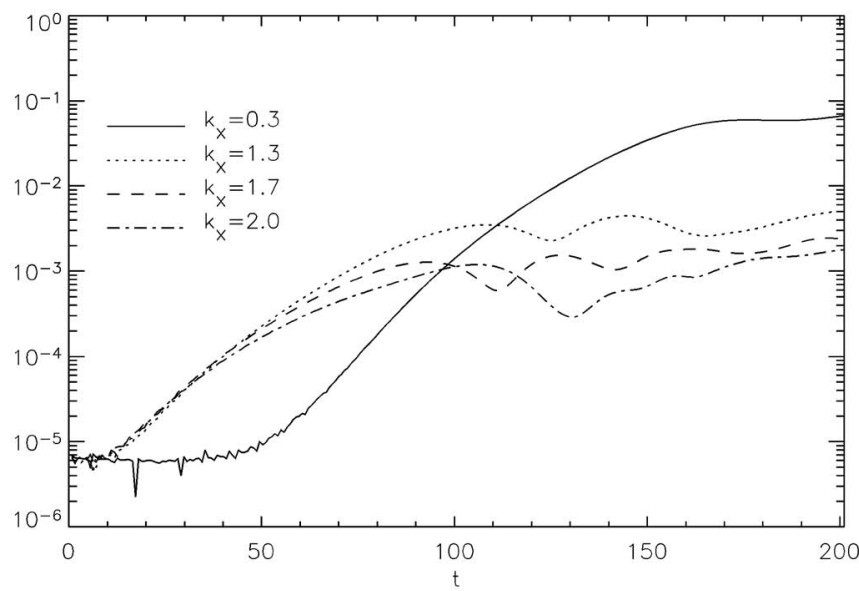

For the C-5, W-7, and W-5 schemes, the measured growth rate of the fluctuating energy is lower than that expected for the tearing instability with $R_{\eta}=5000$ (see Fig. 1), while for the third order WENO the numerical dissipation is so high that the growth rate is larger than that of the instability we intend to simulate. The C-7 scheme, at least for the parameters and the resolution adopted here, does not show any triggering of an instability, the fluctuating magnetic energy being almost constant during the simulation.

As a second test, we have verified the ability of our numerical tool to reproduce well the linear phase of the tearing instability. For this test, we have used the C-7 scheme and $R_{\eta}=5000$. As expected, in a typical simulation the energy contained in each Fourier harmonic grows in time during the first part of the simulation. However, because at finite resistivity the equilibrium also diffuses in time, the modes do not growth exponentially as expected. This is clearly shown in Fig. 3, left panel, where the magnetic energy of the most unstable modes is plotted as a function of time. The data refer to a simulation in which the initial configuration is the FF equilibrium with $\beta=5$. The more unstable modes are in the range predicted by the linear simulations, but their growth is reduced: Because the instability of the tearing mode is strongly dependent on the magnetic field gradient intensity, the growth rate of the single modes is reduced as the time elapses and the equilibrium field diffuses. To compare the results from the linear code with those from the nonlinear one, diffusion of the equilibrium magnetic field must be eliminated. In this case, during the linear phase, each mode grows approximately with a constant slope in logarithmic scale (see the right panel of Fig. 3).

With the equilibrium magnetic field dissipation ruled out, we have performed a set of simulations perturbing a single mode at a time in the instability region of wavenumber space. The corresponding dispersion relation is compared to that obtained by the linear simulations in Fig. 4 for the FF equilibrium and $\beta=5$. There is a small difference between the growth rate values obtained with the linear code and those obtained by the nonlinear code for the most unstable mode, which grows more slowly than predicted by the

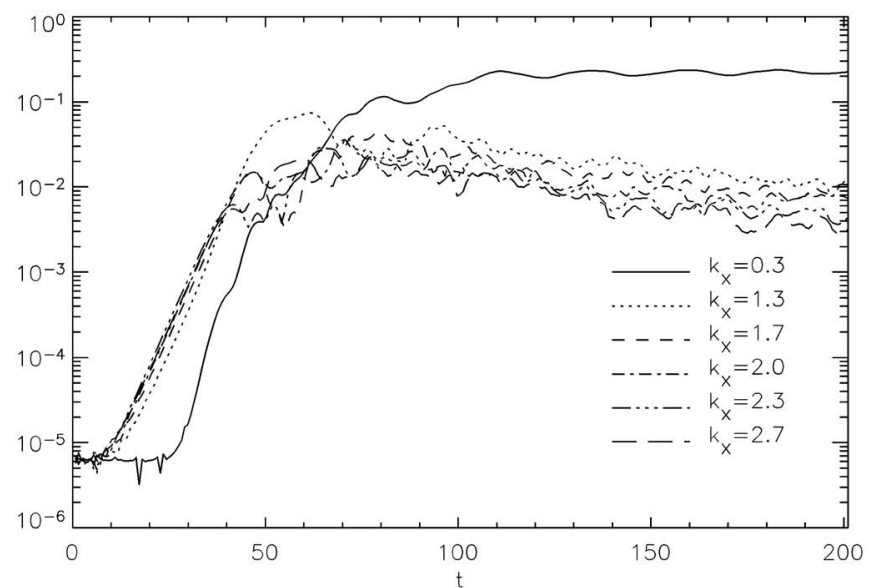

FIG. 3. Magnetic energy time evolution for the most unstable modes and the fundamental mode $k_{x}=0.3$ in typical $2 \mathrm{D}$ simulations. The figure here refers to the force-free case with $\beta=5.0$. In the left panel, the equilibrium magnetic field is allowed to dissipate, while in the right panel, equilibrium dissipation has been ruled out. 


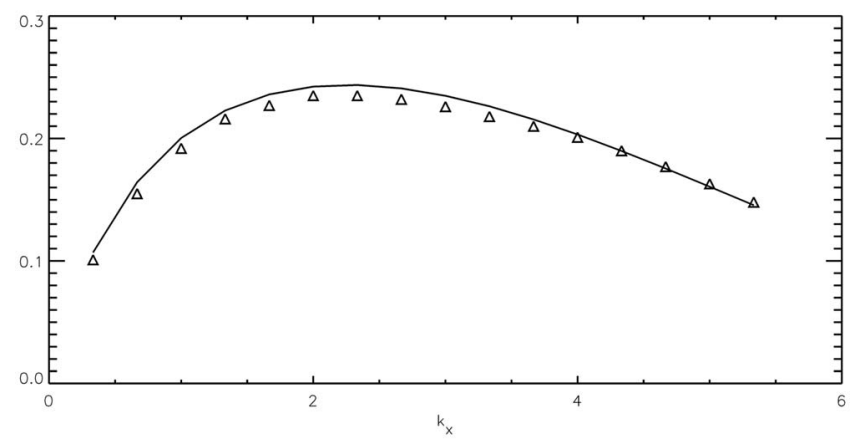

FIG. 4. Comparison between the dispersion relations obtained by the linear simulations (solid line) and the two-dimensional simulations performed by the nonlinear code (triangles). The growth rates are those obtained starting from a force-free configuration and $\beta=5$.

linear code. This can be due to the fact that, despite the smallness of the initial monochromatic perturbation, there is always a nonlinear coupling between modes in the fully nonlinear case.

Before discussing the three-dimensional simulations, we briefly summarize the nonlinear evolution of the $2 \mathrm{D}$ case, which is similar for the three equilibria considered. The nonlinear growth of the unstable modes is followed by the coalescence of the magnetic island created during the linear phase. From the spectral point of view, such coalescence is described by the growth of the power contained in the fundamental mode, which, after $t=100$, overwhelms the power contained in all the other ones. The coalescence is well developed at the end of the simulation. In Fig. 5, we report in the left panel the pressure levels in gray scale and the magnetic field lines at $t=201$. We observe the presence of one magnetic island that is centered at $y=0$ and $x \simeq 5$. It extends along the $x$ direction and it is characterized by an increase in the plasma pressure. The current density intensity shown in the right panel of Fig. 5 shows the presence of a current sheet, which connects the two sides of the magnetic island. A current density enhancement also envelopes the magnetic island.
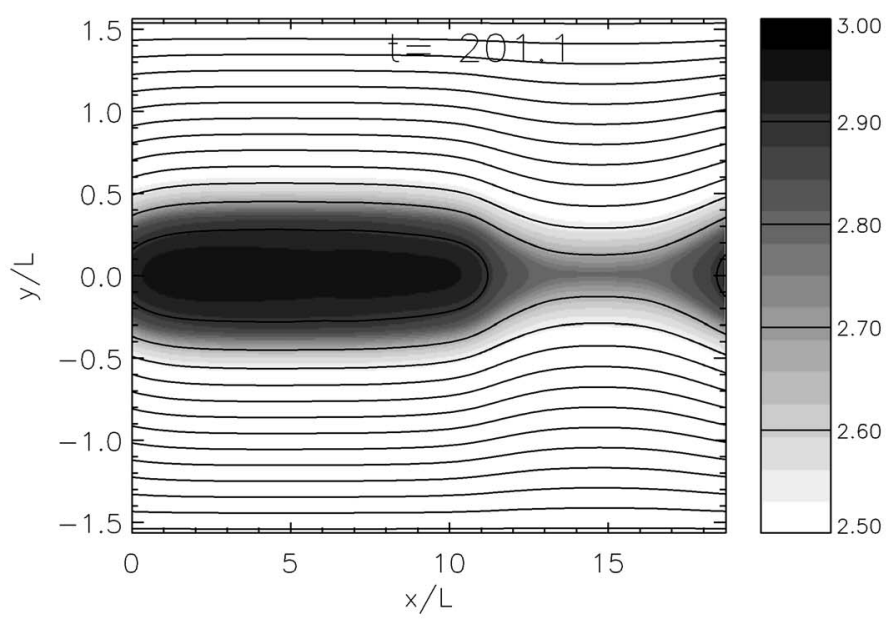

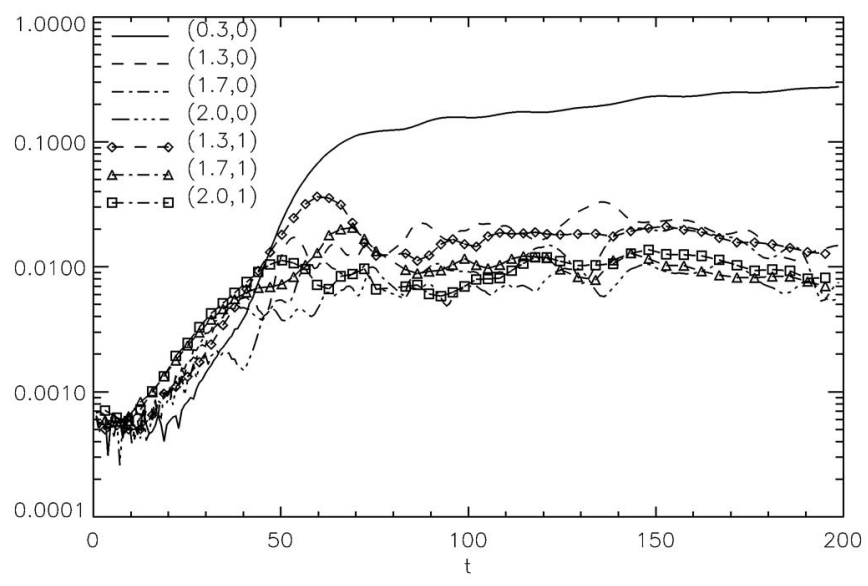

FIG. 6. Magnetic energy spectra evolution for some modes characterized by wave vectors $\left(k_{x}, k_{z}\right)$ for the pressure equilibrium configuration with guide field $B_{0 z}=1$.

\section{THREE-DIMENSIONAL NONLINEAR SIMULATIONS}

In the following subsections, we will discuss in detail results coming from the different magnetic and plasma field initial configurations described previously, namely (a) a pressure equilibrium with a guide field (Sec. VI A), (b) a pressure balanced configuration without a guide field (Sec. VI B), and (c) a force-free equilibrium configuration (Sec. VI C). In each of the following subsections, we discuss the case $\beta=5$. An overview of the results obtained in the low plasma $\beta$ regime is presented in Sec. VI D.

\section{A. Pressure equilibrium with guide field}

The initial evolution of the PE equilibrium configuration with guide field is characterized, after an initial phase $(t$ $<10$ ) of rearrangement of the initial noise, by a linear stage where the unstable modes grow exponentially in time (see Fig. 6). As for the 2D evolution, the most unstable modes are in the range predicted by the linear theory, i.e., with 1.3 $<k_{x}<2.3$. However, in the 3D simulation the most unstable modes are characterized by $k_{z}=1$. In particular, the maximum

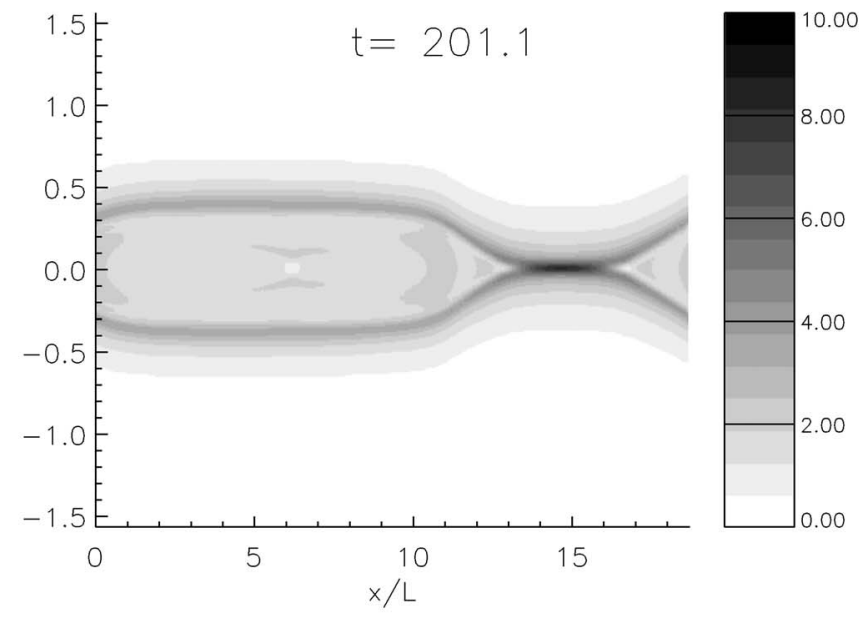

FIG. 5. Left: Pressure intensity (gray scale) and magnetic field lines (black lines) at $t=201$ for a 2D simulation of the tearing instability. The initial configuration was a force-free equilibrium with $\beta=5$. Right: Current density intensity at the same time for the same simulation. 

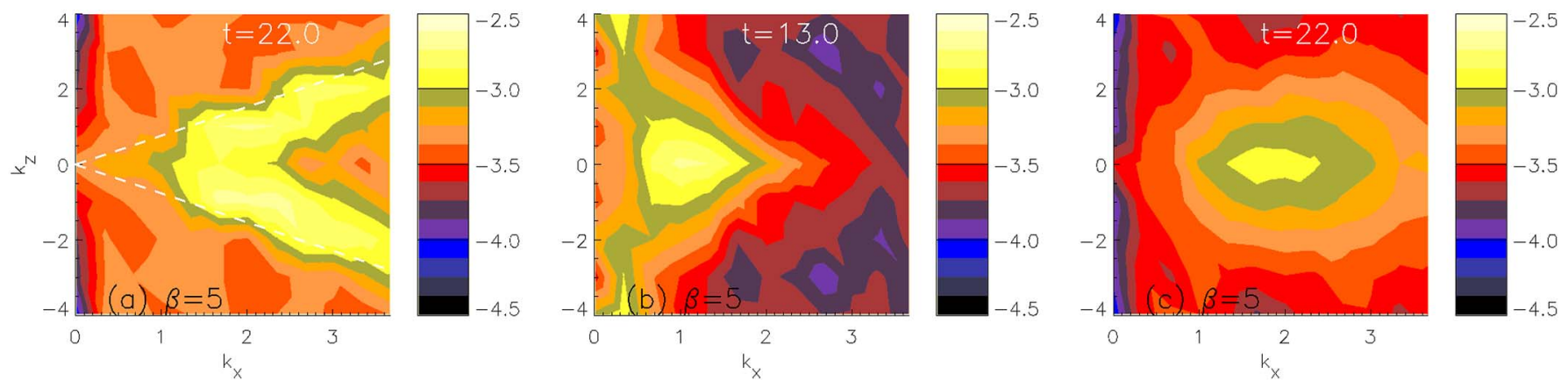

FIG. 7. (Color online) Contour plot of the magnetic energy spectrum in logarithmic scale at a given instant during the linear phase. Panel (a) refers to a simulation with pressure balance configuration and guide field, (b) to the pressure equilibrium configuration without guide field, and (c) to the force-free equilibrium. In (a), the white dashed line highlights the asymptotic direction of the magnetic field in the $(x, z)$ plane.

growth is observed for the modes $\left(k_{x}, k_{z}\right)=(1.7, \pm 1)$, $(2.0, \pm 1)$, and $(2.3, \pm 1)$. This seems to contradict Squire's theorem, ${ }^{39,40}$ which states that for any three-dimensional eigenmode there is a related two-dimensional eigenmode that is more unstable. A similar observation was made by Onofri et $a{ }^{27}{ }^{27}$ in their incompressible simulations, and they suggest that this behavior can be due to nonlinear effects, namely the coupling of the initially excited Fourier harmonics. A twodimensional contour plot of the magnetic energy spectrum in the $\left(k_{x}, k_{z}\right)$ plane during the linear phase for all three cases considered here (Fig. 7) reveals, however, this feature to be peculiar to this initial configuration. A similar behavior has been observed also in 3D simulations of the current-vortex sheet, and it was observed to be related to the angle between the asymptotic magnetic field and the current sheet. ${ }^{41}$ In the $\mathrm{PE}$ with guide field, the asymptotic magnetic field is oriented at $\theta_{B}=\pi / 4$ in the $(x, z)$ plane, which is roughly the direction of the most energetic wave vectors [Fig. 7, panel (a)]. On the contrary, both for the PE and the FF configurations, the asymptotic magnetic field is aligned with the current sheet and the most energetic modes are those characterized by $k_{z}$ $=0$. We will address this issue in more detail in a subsequent paper.

For $t>40$, the most unstable modes start to saturate and, at the same time, the fundamental mode $(0.3,0)$, which corresponds to the maximum wavelength along the $x$ direction allowed by the system, starts to dominate the evolution. During the nonlinear regime, the fundamental mode is still slowly growing while the amplitudes of other modes seem to oscillate around values that are one order of magnitude below the amplitude of the fundamental one. In particular, there is no evidence that $3 \mathrm{D}$ modes, i.e., modes that have $k_{z} \neq 0$, are growing: The $(0,1)$ mode, the longest wavelength in the $z$ direction, shown in Fig. 6 , is not overwhelming the 2D coalescence process.

The fact that the fundamental mode $(0.3,0)$ is the most energetic one in the system corresponds in the physical space to a coalescence of a magnetic island and to the formation of an almost 2D structure. Figure 8 gives a 3D representation of the plasma structure at the end of the simulation, $t=198.7$. The gray-shaded surface encloses the region of space where the plasma pressure (right panel) and the current density intensity (left panel) are above a given threshold, namely 95\% of its maximum for the pressure and, in order to recover more structures, 35\% for the current density. As typical in coalescence process, the pressure reveals the presence of a large quasi-2D structure, here centered approximatively at $L_{x} / 4$ and $L_{y} / 2$, while the current density, although slightly more structured, surrounds the pressure concentration. The maximum of the current density is located at the intersection of the two edges of the enhanced pressure region in a fashion very similar to that observed in the $2 \mathrm{D}$ simulation (see Fig. $5)$.

The observed spectra during the nonlinear stage are anisotropic. This can be revealed by showing the contour plot in logarithmic scale of the magnetic energy spectrum as a function of $k_{x}$ and $k_{z}$ at a given instance. This is done in Fig. 9, panel (a). The magnetic energy spectrum is defined averaging along the $y$ direction, i.e.,

$$
E_{m}\left(k_{x}, k_{z}, t\right)=\frac{1}{4 L_{y}} \int_{-L_{y}}^{L_{y}} B^{2}\left(k_{x}, k_{z}\right) d y .
$$

The energy spectrum is dominated by wave vectors in the $x$ direction. As observed also by Onofri et al., ${ }^{27}$ most of the energy is confined along a given direction, highlighted with the dashed line. The slope of this line is given by the condition $k_{z}= \pm k_{x}$ and corresponds to the resonance condition $\mathbf{k} \cdot \mathbf{B}_{\mathbf{0}}=0$.

\section{B. Pressure equilibrium without guide field}

The exclusion of the magnetic guide-field component along the $z$ direction changes drastically the dynamic of the pressure equilibrium configuration. As observed for the previous case, in the initial phase, after a short interval lasting approximately $10 t$, the $2 \mathrm{D}$ tearing modes start to grow (see Fig. 10). The most unstable modes are still in the range predicted by the linear theory and, contrary to what was observed with the presence of the guide field, the 2D modes with $k_{z}=0$ are harmonics with a higher growth rate than the corresponding modes with $k_{z} \neq 0$. This means that for the present configuration, Squire's theorem still holds.

In a second phase, from $t \simeq 20$ and $t \simeq 40$, the fundamental mode $(1 / 3,0)$ is dominating but, at the same time, nonlinear effects tend to increase the 2D modes characterized by $k_{z} \neq 0$ and $k_{x}=0$. At $t \simeq 50$, these modes have prevailed, first 


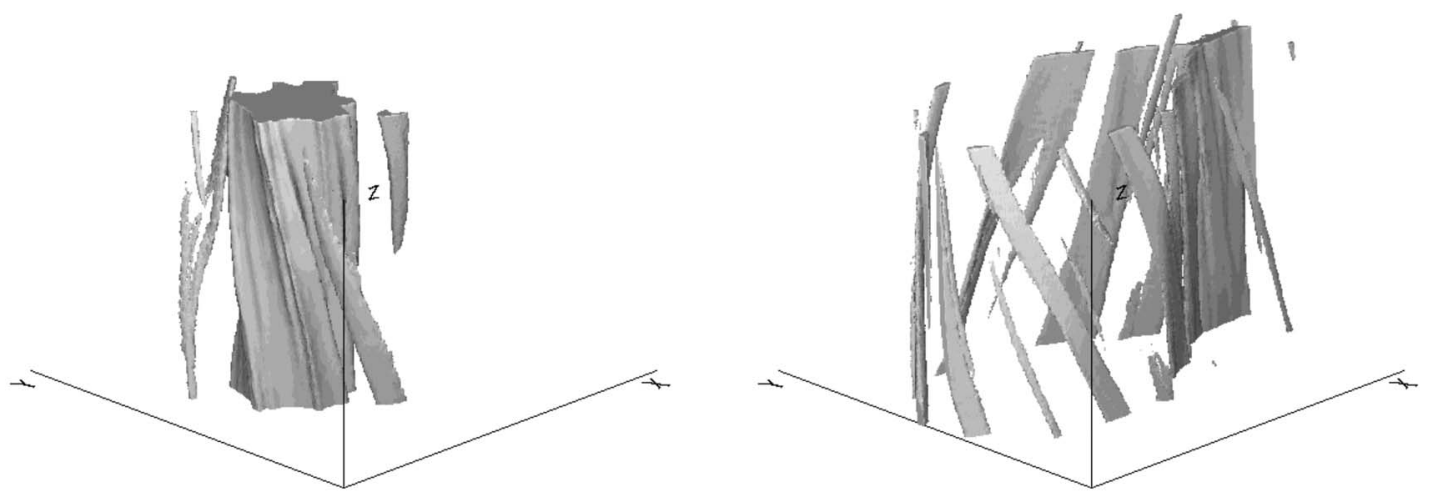

FIG. 8. 3D representation of the plasma and magnetic structure at the end of the simulation $(t=198.7)$ for the case with an initial pressure equilibrium configuration with guide field $B_{z 0}=1$. On the left panel, the gray-shaded surface encloses the region of space where the plasma pressure $p$ exceeds a given threshold, namely $95 \%$ of its maximum value $\left(P_{\max }=2.94\right)$. On the right panel, a similar figure is reported for the current density intensity $J$. To discern more structures, the threshold for $J$ has been set equal to $35 \%$ of its maximum value.

with the mode $(0,2)$ and then with the mode $(0,1)$, the longest wavelength available along the $z$ direction.

In physical space, the behavior observed in the Fourier space corresponds to two processes. In the first stage, the tearing instability leads to the building of magnetic islands that coalesce afterward. At $t \simeq 50$, as shown in Fig. 11, top panel, we observe the presence of a single magnetic island, elongated along the $x$ direction with a current density concentration in the two sides of the magnetic island. At this time, the growth of the $(1 / 3,0)$ mode is stopped and later it is overwhelmed by the mode $(0,2)$. The secondary instability is now dominating the dynamical evolution of the system and the magnetic island bends out of shape. At $t \simeq 150$, the secondary instability has become large enough that the magnetic island is no longer present. In the final state, the system has recovered a basically 2D configuration, but now in the plane $(y, z)$ rather than in the plane $(x, y)$ as at the end of the coalescence process.

This behavior is reflected in the magnetic energy spectrum observed during the nonlinear regime, as reported in Fig. 9, panel (b). The spectrum strongly differs from those observed for the pressure equilibrium configuration with the guide field. Now, most of the energy is contained in modes whose wave vectors are basically oriented along the $z$ direction, confirming that the system is returned toward a new $2 \mathrm{D}$ configuration in the plane $(y, z)$.

\section{Force-free configuration}

In the FF configuration, the basic state does not contain a neutral sheet, as in the PE case, but the field rotates through the current sheet to invert its polarity. In this case, an axial field $B_{z}(y)$, analogous to the guide field of the PE configuration, is present only inside the current sheet. As for the previous simulations, the first part of the simulation is characterized by a rapid rearrangement of the initial perturbations (see Fig. 12). During the linear phase, the dynamic is dominated by the presence of modes characterized by $k_{z}=0$. In particular, we observe that the fastest modes are those observed in the two-dimensional simulation as described in Sec. V, with wave vectors $k_{x}$ in the range 1.3-2.0. This means that also in this case, Squire's theorem still holds.

For $t>30$, the system enters in the nonlinear regime characterized by the growth of the mode $(0.3,0)$ suggesting that the system is following the typical 2D nonlinear evolution with the formation of magnetic island followed by their coalescence. A snapshot of the plasma pressure and of the current density at $t=123$, as shown in the top panels of Fig. 13 , reveals the presence of an almost $2 \mathrm{D}$ structure characterized by a pressure enhancement in the same way as seen in Fig. 8. With respect to the pressure equilibrium configuration with guide field, we observe, however, that the system is significantly modulated along the $z$ direction, revealing that
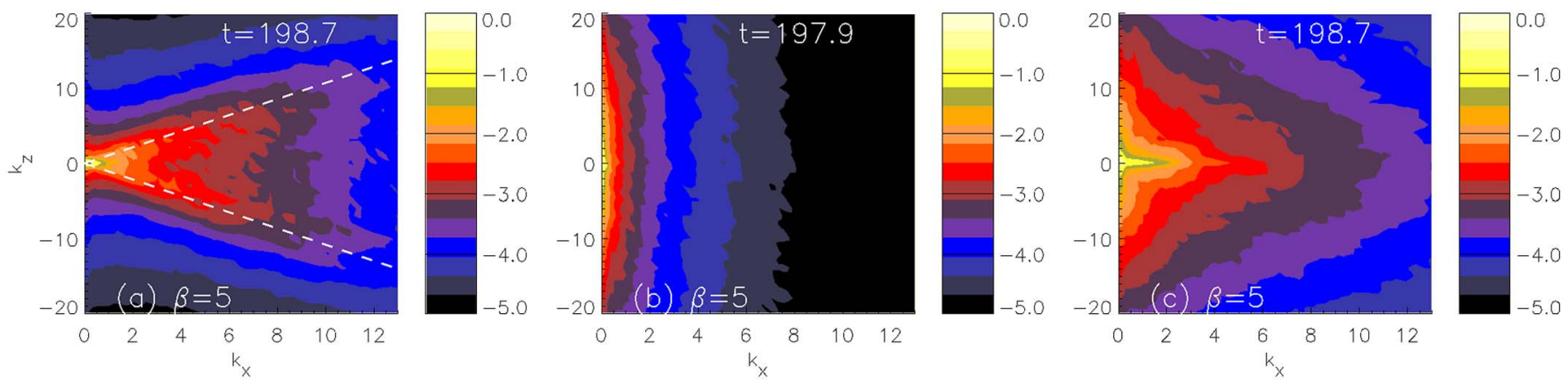

FIG. 9. (Color online) Contour plot of the magnetic energy spectrum in logarithmic scale at a given instant during the nonlinear phase. Panel (a) refers to a simulation with pressure balance configuration and guide field, (b) to the pressure equilibrium configuration without guide field, and (c) to the force-free equilibrium. In (a), the white dashed line highlights the resonant condition $\mathbf{k} \cdot \mathbf{B}_{\mathbf{0}}=0$. 


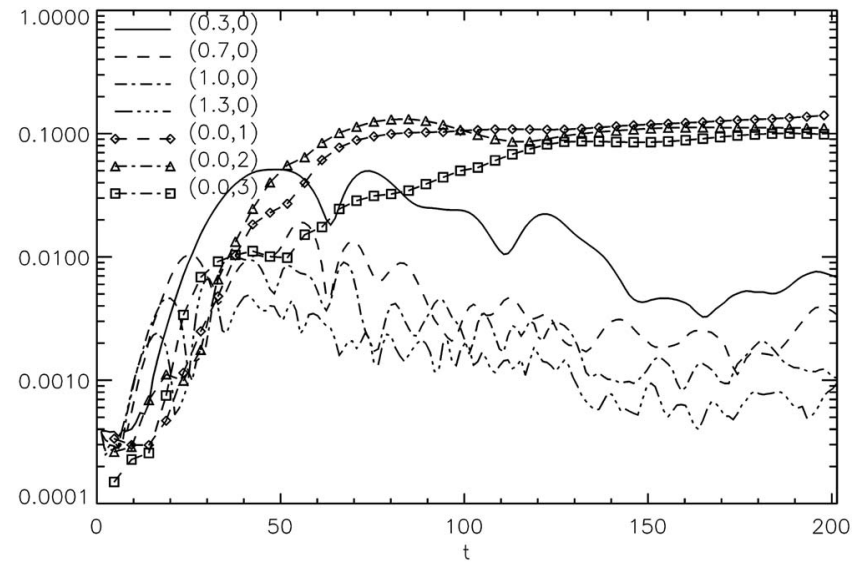

FIG. 10. Same as Fig. 6 for the case of pressure equilibrium configuration without guide field.

the structure is now under the influence of modes characterized by $k_{z} \neq 0$. From Fig. 12 , it appears clearly that the $(0,1)$ mode is still growing during the first part of the nonlinear regime and it overwhelms the fundamental mode after $t$ $=130$. As shown in the middle panels of Fig. 13, at $t=148$ the quasi-2D structure is kinking and at the end of the simulation ( $t=198$, bottom panel of Fig. 13) it is completely destroyed. However, differently from the PE equilibrium without guide field, at the end of the simulation we do not observe any clear evidence of $2 \mathrm{D}$ structuring in the $(y, z)$ plane.

A snapshot of the 2D spectrum at $t=198$, as shown in Fig. 9, panel (c), shows that the system is under the influence of $2 \mathrm{D}$ modes. The first are characterized by $\left(k_{x} \neq 0, k_{z}=0\right)$,
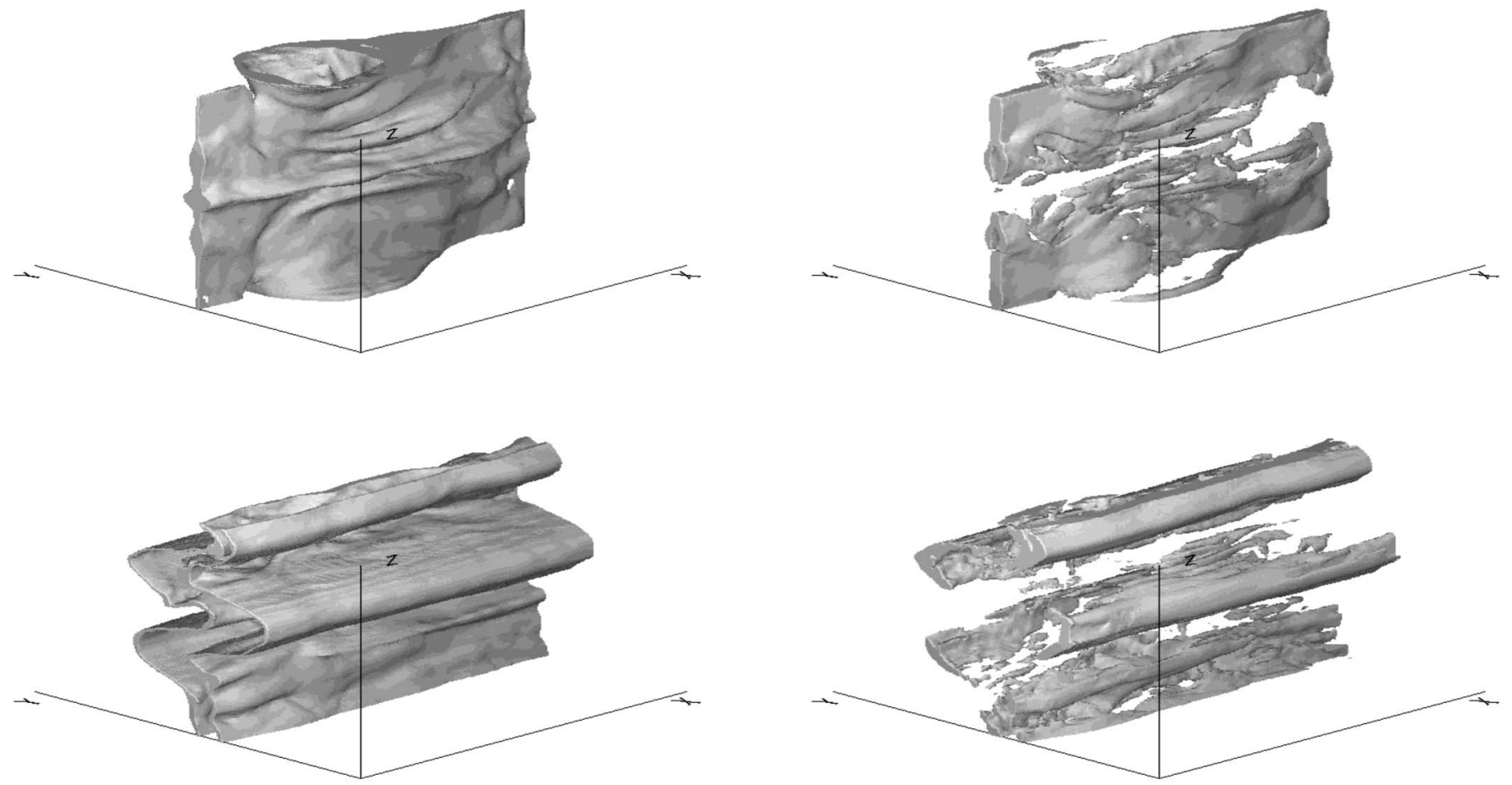

FIG. 11. 3D representation of the plasma and magnetic structure at different times for the simulation with an initial pressure equilibrium configuration without guide field. In the left panels, isosurfaces for the pressure at $p=2.88$ correspond to $97 \%$ of its maximum value. In the right panels, the gray-shaded surface encloses the region of space where the current density is larger than $30 \%$ of its maximum value. Top panel: $t=50$. Bottom panel: $t=150$. 

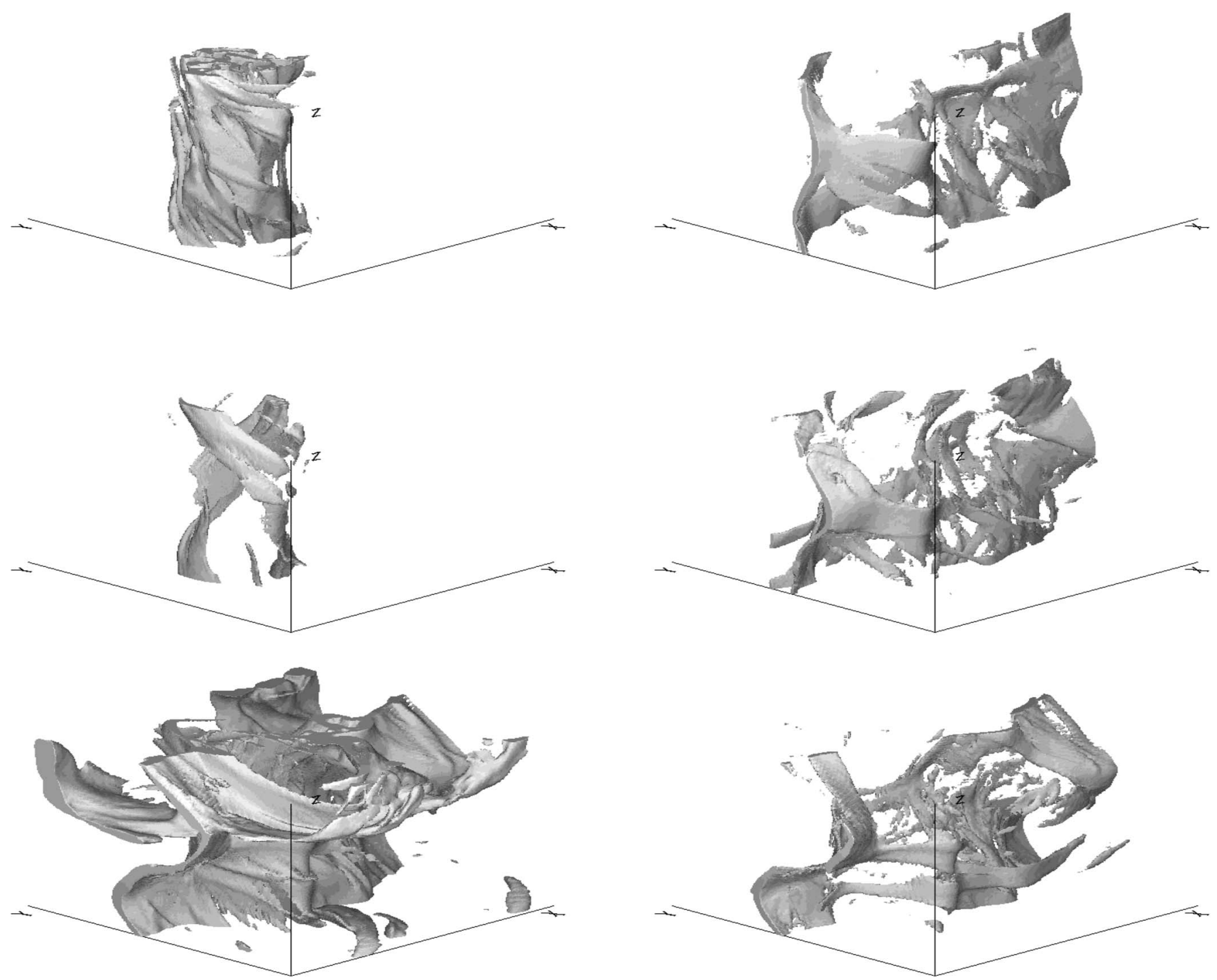

FIG. 13. Top: 3D representation of the plasma and magnetic structure at $t=123$ for the simulation with an initial force-free equilibrium configuration. In the left panel, isosurface for the pressure with value $p=2.85$ corresponding to $97 \%$ of its maximum value. In the right panel, the isosurface of the current density at $40 \%$ of its maximum value at the same instant. As for the previous panels, higher values of pressure and current density are enclosed by these isosurfaces. Middle: Same quantities at $t=148$. Bottom: Same quantities at $t=198$.

$\beta=0.2$ show that the nonlinear evolution of the current sheet seems to be independent of the plasma $\beta$ as well. Figure 14 gives an overview of the plasma pressure for the three simulations at $t=150$. We note that the pressure equilibrium con- figuration in the presence of the guide field has developed a large quasi-2D structure as seen for the high $\beta$ regime. Similarly, the magnetic energy spectrum, shown in Fig. 15, panel (a), is highly anisotropic, showing a preferential direction
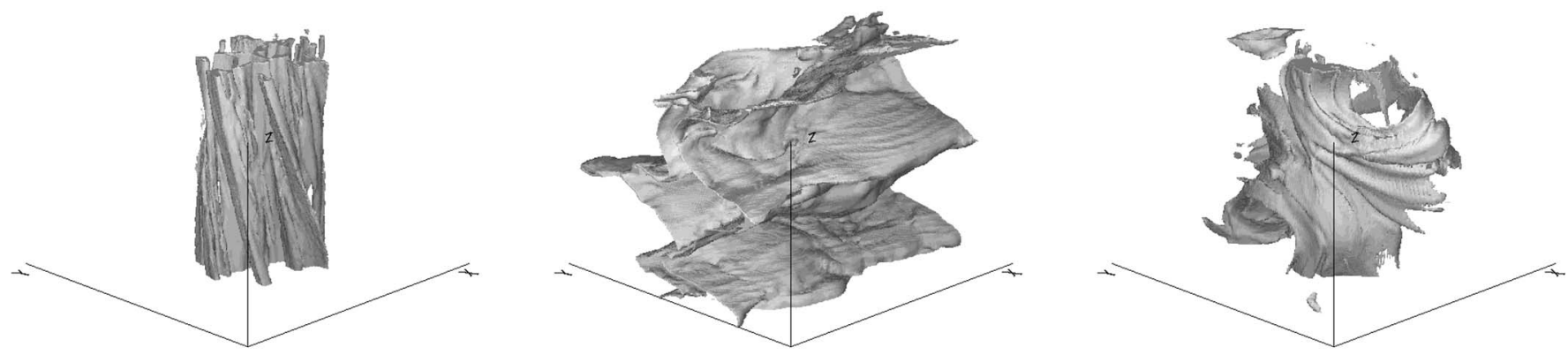

FIG. 14. 3D representation of the plasma structure for three simulations with $\beta=0.2$ : pressure equilibrium with guide field (left), pressure equilibrium without guide field (middle), and force-free configuration (left). For all the simulations, isosurfaces are represented for the pressure at $t=150: p=0.48$ for the pressure equilibrium with guide field, $p=0.45$ for pressure equilibrium, and $p=0.41$ for the force-free configuration. 

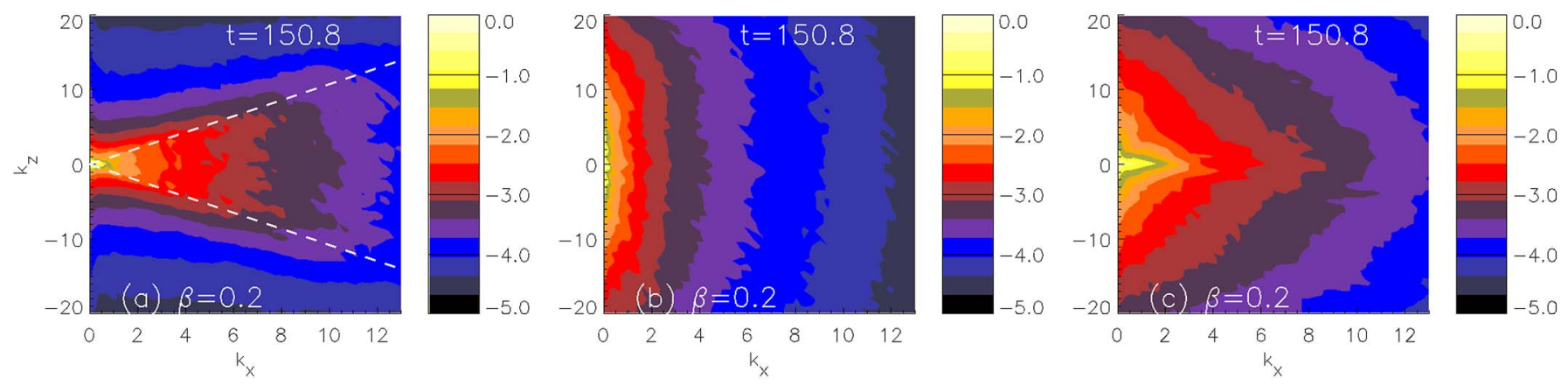

FIG. 15. (Color online) Same as Fig. 9 for the low beta simulations $\beta=0.2$.

corresponding to the resonant condition in a fashion very similar to that observed for the corresponding high $\beta$ simulation. The same conclusion can be inferred for the pressure equilibrium configuration without guide field: The nonlinear regime is dominated here by quasi-2D structures lying in the $(y, z)$ plane, and the magnetic energy is mainly contained by modes whose wave vectors are basically oriented along the $z$ direction [see Fig. 15, panel (b)]. The force-free configuration at $t=150$ turns out to be more structured with the presence of a quasi-2D structure in the $(x, y)$ plane strongly modulated by the presence of modes directed along the $z$ direction. The magnetic energy spectrum reported in Fig. 15, panel (c), appears to be essentially the same as that shown for the corresponding $\beta=5$ simulation.

\section{DISCUSSION AND CONCLUSIONS}

We have followed the 3D linear and nonlinear evolution of the tearing instability for different initial configurations of the current sheet in the framework of compressible and resistive MHD.

Numerical simulations have revealed that the fate of the current sheet in the late stage of its evolution is strongly affected by the initial configuration. For a basic state characterized by a pressure equilibrium configuration without a guide field, the 1D initial configuration is driven by the tearing instability toward a 2D structure characterized by magnetic islands that tend to coalesce. However, as observed by Dahlburg and collaborators, ${ }^{16,18}$ the 2D structure is destabilized by a secondary instability that tends to destroy the basic state through a three-dimensional instability. At later stages, the system recovers a new 2D configuration essentially oriented in the plane perpendicular to the initial current sheet [the $(y, z)$ plane in our simulations]. Such behavior is reflected in the magnetic energy spectrum, which appears to be strongly anisotropic, with most of its energy contained in modes whose wave vectors are basically aligned with the $z$ direction.

As conjectured by Dahlburg and Einaudi ${ }^{18}$ and shown numerically by Onofri et al. ${ }^{27}$ the presence of a guide field, i.e., of a homogeneous magnetic field aligned with the current density vector, has a stabilizing effect for the developing of the secondary instability. Our numerical simulations confirm that this is true also in the compressible case, independently of the plasma beta adopted: the nonlinear evolution of such a configuration leads to the formation of magnetic is- lands that coalesce afterward. We do not see any indication of the starting of a secondary instability. The spectral evolution of the system turns out to be very different from that observed in the case in which the guide field was absent. The energy magnetic spectrum is still anisotropic but with a preferential direction that is not the $x$ nor the $z$ direction but is given by the resonant condition $\mathbf{k} \cdot \mathbf{B}_{\mathbf{0}}=0$. Most of the magnetic energy is in the modes that satisfy such a condition and in the region of space where $k_{x}>k_{z}$. The outer region of the wave-vector space results is deprived of magnetic energy. A similar behavior was also observed by Onofri et al., ${ }^{27}$ although in their simulations, an enlargement was observed of the anisotropy angle, which has not been observed in our simulations. One reason could be the fact that our simulations stop earlier than those of Onofri and collaborators. It may be noticed, however, that we start our simulations with a much higher level of initial disturbances so that the linear stage is much shorter, the magnetic energy contained in the fluctuations at the end of our simulations being of the same order as that obtained by Onofri et al. at much later instances. Dahlburg et al. $^{28}$ have studied the growth rate of the secondary instability as a function of the guide-field intensity in the incompressible limit. Their study shows that the secondary instability is stopped if the guide-field intensity is beyond a given threshold, which depends on the Lundquist number. Our compressible simulations are consistent with their results: For the Lundquist number adopted here and for the wavelengths allowed by our system, Dahlburg et al. ${ }^{28}$ predict the system to be stable to the secondary instability.

The fate of the current sheet created by a force-free configuration, to our knowledge not yet studied in its 3D nonlinear evolution, is even different from those where the equilibrium is ensured by a pressure equilibrium. In this case, the magnetic field is, by definition, always aligned with the current density vector. The important difference with respect to the pressure equilibrium configuration with a guide field is that now the magnetic field component parallel to the current density is concentrated only in the current sheet. Its nonlinear dynamic reveals that the system follows an intermediate path between the two previous cases. In the first part, the system is characterized by the typical 2D nonlinear evolution of the tearing instability with the formation of a chain of magnetic islands that afterward tend to coalesce. Successively, the structure is destabilized by 3D modes that kink the system and destroy the quasi-2D structure. At the end of the 
TABLE I. Coefficients for the REC procedures available in ECHO.

\begin{tabular}{lccccccccc}
\hline \hline REC & $\alpha$ & $\beta$ & $r_{-3}$ & $r_{-2}$ & $r_{-1}$ & $r_{0}$ & $r_{1}$ & $r_{2}$ & $r_{3}$ \\
\hline E3 & $\ldots$ & $\ldots$ & $\ldots$ & $\ldots$ & $-1 / 8$ & $3 / 4$ & $3 / 8$ & $\ldots$ & $\ldots$ \\
E5 & $\ldots$ & $\ldots$ & $\ldots$ & $3 / 128$ & $-20 / 128$ & $90 / 128$ & $60 / 128$ & $-5 / 128$ & $\ldots$ \\
E7 & $\ldots$ & $\ldots$ & $-5 / 1024$ & $21 / 512$ & $-175 / 1024$ & $175 / 256$ & $525 / 1024$ & $-35 / 512$ & $7 / 1024$ \\
C5 & $1 / 2$ & $1 / 10$ & $\ldots$ & $\ldots$ & $1 / 10$ & 1 & $1 / 2$ & $\ldots$ & $\cdots$ \\
C7 & $1 / 2$ & $3 / 14$ & $\ldots$ & $-1 / 224$ & $1 / 8$ & $15 / 16$ & $5 / 8$ & $1 / 32$ & $\cdots$ \\
\hline \hline
\end{tabular}

simulation, the system is characterized by the presence of a large dose of modes whose wave vectors are directed along the $z$ direction. A significant fraction of the energy is also contained in modes characterized by wave vectors directed along the $x$ direction (the original direction of the current sheet). The corresponding spectrum is anisotropic with two preferential directions as reservoirs of the fluctuating energy. The role of the plasma pressure with respect the magnetic field pressure seems to be negligible in the linear and the subsequent nonlinear evolution of the tearing instability at least with regard to the growth rate of the primary instability and the insurgence of the secondary instability. For the three initial magnetic field configurations adopted here, both the magnetic structure and the magnetic energy spectrum have not revealed any significant difference between the low and high $\beta$ regime.

It has been shown that Hall physics is an important ingredient in the development of fast reconnection ${ }^{42-52}$ and can have an influence on the linear and nonlinear evolution of the tearing instability. ${ }^{42,53,54}$ Future work will include the Hall effect in studying the dynamical evolution of the current sheet. The current sheet evolution has been investigated here in the limit of zero kinematic viscosity. It has been suggested, however, that the Hartmann number, which is the geometric mean of the kinetic and magnetic Reynolds number, is the essential parameter that determines the global stability properties of the plane sheet pinch. ${ }^{55,56}$

\section{ACKNOWLEDGMENTS}

The authors are grateful to Luca Del Zanna for his useful comments and suggestions. Numerical computations were performed using the 512 processor IBM SP5 of the CINECA consortium available through the INAF-CINECA agreement 2006-2007: High Performance Computing Resources for Astronomy and Astrophysics.

This work was carried out by the Jet Propulsion Laboratory under contract from NASA through the Living with a Star and Science, Research \& Technology programs.

\section{APPENDIX: IMPLICIT RECONSTRUCTION- DERIVATION PROCEDURES}

The reconstruction operation (REC) employed in ECHO is based on piecewise polynomial interpolation that can be both explicit and implicit.

Given a uniform stencil of $N$ cell-centered grid points $\left\{x_{i}\right\}$ of interval $\Delta x$ with corresponding values $\left\{f_{i}\right\}$ of the discretized function $f(x)$, the scope is to find an $r$ th order ap- proximation of the function $f_{i+1 / 2}$ at the interface $x_{i+1 / 2}$. Contrary to intercell interpolation, the upwind procedures are based on either left biased (L) and right biased (R) stencils. In explicit interpolation routines, an odd number $r$ of grid points symmetric to $x_{i}$ and to $x_{i+1}$ for left and right biased interpolation are used, respectively, which give an interpolated value of the function at $x_{i+1 / 2}$ accurate up to the $r$ th order. In the implicit REC procedure, the left biased interpolation is obtained by an approximation of the form

$$
f_{i+1 / 2}^{L}+\sum_{n=1}^{l}\left(\alpha_{n} f_{i+1 / 2-n}^{L}+\beta_{n} f_{i+1 / 2+n}^{L}\right)=\sum_{n=1}^{m} r_{n-(m+1) / 2} f_{i-(m+1) / 2+n}
$$

where, as for the explicit interpolation, an odd number $m$ of cell-centered values of $f_{i}$ symmetric to $x_{i}$ is used on the rhs, and an odd number $2 l+1$ of reconstructed function $f_{i+1 / 2}^{L}$ values symmetric to $x_{i+1 / 2}$ appears on the lhs. For the R-states, the rhs of Eq. (A1) will be symmetric to $x_{i+1}$ and the lhs is left unchanged. In the general case, the maximum order of the reconstruction is $r=2 l+m$. The coefficients $\alpha_{n}, \beta_{n}$, and $r_{n-(m+1) / 2}$ are obtained by matching the Taylor series coefficients of various orders after expansion around $x_{i+1 / 2}$ of the function $f$. In ECHO, we may use explicit third (E3), fifth (E5), and seventh (E7) or compact fifth (C5) and seventh (C7) order reconstruction algorithms. For the compact algorithms, we use $l=1$, which requires numerically the inversion of a tridiagonal matrix. The coefficient values of each reconstruction procedure used in ECHO are reported in Table I.

The spectral-like resolution properties of the above procedures can be studied with a Fourier analysis of the errors associated with the approximation introduced. ${ }^{30}$ For this scope, the function $f$ is considered to be periodic in the domain $\left[0, x_{M}\right]$ with $\Delta x=x_{M} / N$ so that $f$ may be decomposed into its Fourier coefficients,

$$
f(x)=\sum_{k=-N / 2}^{k=N / 2} \tilde{f}_{k} \exp \left(j \frac{2 \pi k x}{x_{M}}\right)
$$

with $j=\sqrt{-1}$. Introducing the normalized wave vector $w$ $=2 \pi k \Delta x / x_{M}$ and the normalized variable $s=x / \Delta x$, Fourier modes are simply $\exp (j w s)$ and the normalized wave vectors are in the range $[0, \pi]$. Applying Eq. (A2) and the above normalizations to Eq. (A1), we have 


$$
\begin{aligned}
\tilde{f}_{w} \mathrm{e}^{j w s}\left[1+\sum_{n=1}^{l}\left(\alpha_{n} \mathrm{e}^{-j w n}+\beta_{n} \mathrm{e}^{j w n}\right)\right] \\
=\widetilde{f}_{w} \mathrm{e}^{j w s} \sum_{n=1}^{m} r_{k-(m+1) / 2} \mathrm{e}^{j[2(n-1)-m] w / 2} .
\end{aligned}
$$

Defining the transfer function as

$$
T(w)=\frac{\sum_{n=1}^{m} r_{k-(m+1) / 2} \mathrm{e}^{\mathrm{j}[2(n-1)-m] w / 2}}{1+\sum_{n=1}^{l}\left(\alpha_{n} \mathrm{e}^{-j w n}+\beta_{n} \mathrm{e}^{j w n}\right)},
$$

for an exact reconstruction we should have $T(w)=1$. The range of wave numbers over which the condition $T(w)=1$ holds within a specified error tolerance defines the set of well-resolved waves.

The high-order approximation of the flux function at the cell center involves the derivation (DER) operation. Given the intercell flux values $\left\{f_{i+1 / 2}\right\}$, the scope is to find a numerical flux function $\hat{f}_{i+1 / 2}$ such that the flux difference approximates the derivative $f^{\prime}(x)$ to the desired order. In the explicit DER operation, this means

$$
\Delta x f_{i}^{\prime} \simeq \hat{f}_{i+1 / 2}-\hat{f}_{i-1 / 2}=\sum_{n=1}^{m} \frac{d_{n}}{2 n-1}\left[f_{i+(n-1 / 2)}-f_{i-(n-1 / 2)}\right],
$$

which gives accuracy up to the $2 m$ th order. For the implicit DER, we use

$$
\begin{aligned}
{\left[f_{i}^{\prime}+\sum_{n=1}^{l} \alpha_{n}\left(f_{i-n}^{\prime}+f_{i+n}^{\prime}\right)\right] \Delta x=} & \sum_{n=1}^{m} \frac{d_{n}}{2 n-1}\left[f_{i+(n-1 / 2)}\right. \\
& \left.-f_{i-(n-1 / 2)}\right] .
\end{aligned}
$$

The accuracy is $2 m+2 l$. ECHO is able to handle different DER procedures: Explicit fourth (E4), sixth (E6), and eighth (E8) or compact sixth (C6) and eighth (C8) order algorithms. Coefficient values for each DER are listed in Table II. As for the REC, compact DER used in ECHO are limited to the case $l=1$.

The spectral properties of the DER operation can be analyzed by using the transfer function in the same way shown for the REC operation. Applying Eq. (A2) to Eq. (A6), we have

TABLE II. Coefficients for the DER procedures available in ECHO.

\begin{tabular}{lccccc}
\hline \hline DER & $\alpha$ & $d_{1}$ & $d_{2}$ & $d_{3}$ & $d_{4}$ \\
\hline E4 & $\ldots$ & $9 / 8$ & $-1 / 8$ & $\ldots$ & $\ldots$ \\
E6 & $\ldots$ & $75 / 64$ & $-25 / 128$ & $3 / 128$ & $\ldots$ \\
E8 & $\ldots$ & $1225 / 1024$ & $-245 / 1024$ & $49 / 1024$ & $-5 / 1024$ \\
C6 & $9 / 62$ & $63 / 62$ & $17 / 62$ & $\ldots$ & $\ldots$ \\
C8 & $25 / 118$ & $2675 / 2832$ & $925 / 1888$ & $-61 / 5664$ & $\ldots$ \\
\hline \hline
\end{tabular}
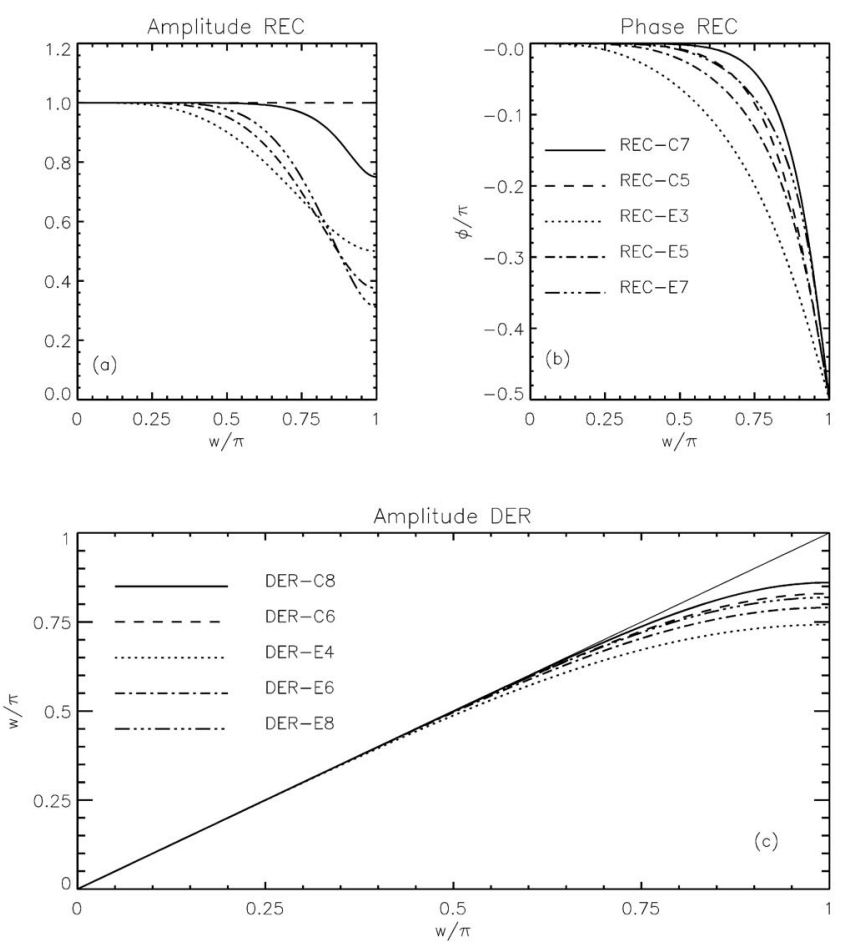

FIG. 16. Transfer function for different REC and DER operations. (a) and (b) Amplitude and phase of the transfer function Eq. (A4) for the REC operations listed in Table I. (c) Transfer function Eq. (A8) for the DER operation listed in Table II.

$$
\begin{aligned}
& w \widetilde{f}_{w} \mathrm{e}^{j w s}\left[1+2 \sum_{n=1}^{l} \alpha_{n} \cos (n w)\right] \\
& =2 \tilde{f}_{w} \mathrm{e}^{j w s} \sum_{n=1}^{m} \frac{d_{n}}{2 n-1} \sin [(n-1 / 2) w] .
\end{aligned}
$$

Defining the transfer function for the DER operation as

$$
T(w)=\frac{2 \sum_{n=1}^{m} \frac{d_{n}}{2 n-1} \sin [(n-1 / 2) w]}{1+2 \sum_{n=1}^{l} \alpha_{n} \cos (n w)},
$$

we should have $T(w)=w$ for an exact spectral resolution for the DER operation.

A comparison of the spectral resolution properties of some REC and DER procedures is shown in Fig. 16, where the module and phase of transfer functions given by Eqs. (A4) and (A8) are plotted. We note in general a better spectral resolution for compact procedures with respect to explicit ones having the same numerical accuracy. The higherorder compact schemes (REC-C7 and DER-C8) give a good spectral resolution up to $3 / 4$ of the maximum allowed wave number.

${ }^{1}$ L. G. Bagalá, C. H. Mandrini, M. G. Rovira, and P. Démoulin, Astron. Astrophys. 363, 779 (2000).

${ }^{2}$ C. H. Mandrini, P. Démoulin, and J. A. Klimchuk, Astrophys. J. 530, 999 (2000).

${ }^{3}$ D. Biskamp, Nonlinear Magnetohydrodynamics, 1st ed. (Cambridge University Press, Cambridge, 1993).

${ }^{4}$ H. P. Furth, J. Killeen, and M. N. Rosenbluth, Phys. Fluids 6, 459 (1963).

${ }^{5}$ J. Wesson, Nucl. Fusion 6, 130 (1966). 
${ }^{6}$ R. S. Steinolfson and G. van Hoven, Phys. Fluids 26, 117 (1983).

${ }^{7}$ A. Bhattacharjee, F. Brunel, and T. Tajima, Phys. Fluids 26, 3332 (1983).

${ }^{8}$ D. Biskamp and H. Welter, Phys. Rev. Lett. 44, 1069 (1980).

${ }^{9}$ A. Bondeson, Phys. Fluids 26, 1275 (1983).

${ }^{10}$ J. M. Finn and P. K. Kaw, Phys. Fluids 20, 72 (1977).

${ }^{11}$ P. L. Pritchett and C. C. Wu, Phys. Fluids 22, 2140 (1979).

${ }^{12}$ T. Hayashi, J. Phys. Soc. Jpn. 50, 3124 (1981).

${ }^{13}$ F. Malara, P. Veltri, and V. Carbone, Phys. Fluids B 3, 1801 (1991).

${ }^{14}$ F. Malara, P. Veltri, and V. Carbone, Phys. Fluids B 4, 3070 (1992).

${ }^{15}$ R. B. Dahlburg, J. Plasma Phys. 57, 35 (1997).

${ }^{16}$ R. B. Dahlburg, S. K. Antiochos, and T. A. Zang, Phys. Fluids B 4, 3902 (1992).

${ }^{17}$ R. B. Dahlburg and G. Einaudi, Phys. Plasmas 8, 2700 (2001).

${ }^{18}$ R. B. Dahlburg and G. Einaudi, Phys. Lett. A 294, 101 (2002).

${ }^{19}$ R. W. Metcalfe, S. A. Orszag, M. E. Brachet, S. Menon, and J. J. Riley, J. Fluid Mech. 184, 207 (1987).

${ }^{20}$ S. A. Orszag and A. T. Patera, J. Fluid Mech. 128, 347 (1983).

${ }^{21}$ J. Schumacher and N. Seehafer, Phys. Rev. E 61, 2695 (2000).

${ }^{22}$ R. B. Dahlburg and J. T. Karpen, Astrophys. J. 434, 766 (1994).

${ }^{23}$ G. Einaudi, P. Boncinelli, R. B. Dahlburg, and J. T. Karpen, J. Geophys. Res. 104, 521, DOI: 10.1029/98JA02394 (1999).

${ }^{24}$ L. Bettarini, S. Landi, F. A. Rappazzo, M. Velli, and M. Opher, Astron. Astrophys. 452, 321 (2006).

${ }^{25}$ R. B. Dahlburg, P. Boncinelli, and G. Einaudi, Phys. Plasmas 5, 79 (1998).

${ }^{26}$ R. B. Dahlburg and G. Einaudi, Phys. Plasmas 7, 1356 (2000).

${ }^{27}$ M. Onofri, L. Primavera, F. Malara, and P. Veltri, Phys. Plasmas 11, 4837 (2004).

${ }^{28}$ R. B. Dahlburg, J. A. Klimchuk, and S. K. Antiochos, Astrophys. J. 622, 1191 (2005).

${ }^{29}$ E. J. Smith, J. Geophys. Res. 106, 15819, DOI: 10.1029/2000JA000120 (2001).

${ }^{30}$ S. K. Lele, J. Comput. Phys. 103, 16 (1992).

${ }^{31}$ P. Londrillo and L. Del Zanna, Astrophys. J. 530, 508 (2000).

${ }^{32}$ P. Londrillo and L. Del Zanna, J. Comput. Phys. 195, 17 (2004).

${ }^{33}$ L. Del Zanna, N. Bucciantini, and P. Londrillo, Astron. Astrophys. 400, 397 (2003)
${ }^{34}$ L. Del Zanna, O. Zanotti, N. Bucciantini, and P. Londrillo, Astron. Astrophys. 473, 11 (2007).

${ }^{35}$ X.-D. Liu, S. Osher, and T. Chan, J. Comput. Phys. 115, 200 (1994).

${ }^{36}$ A. Suresh and H. T. Huynh, J. Comput. Phys. 136, 83 (1997).

${ }^{37}$ C.-W. Shu and S. Osher, J. Comput. Phys. 77, 439 (1988).

${ }^{38}$ S. Landi, M. Velli, and G. Einaudi, Astrophys. J. 624, 392 (2005).

${ }^{39}$ R. B. Dahlburg and J. T. Karpen, J. Geophys. Res. 100, 23489, DOI: 10.1029/95JA02496 (1995).

${ }^{40}$ N. Seehafer and J. Schumacher, Phys. Plasmas 4, 4447 (1997).

${ }^{41}$ L. Bettarini, S. Landi, M. Velli, and P. Londrillo, "Magnetic and velocity shear driven instabilities in the heliospheric plasma," Surveys Geophys. (submitted).

${ }^{42}$ J. Birn, J. F. Drake, M. A. Shay, B. N. Rogers, R. E. Denton, M. Hesse, M. Kuznetsova, Z. W. Ma, A. Bhattacharjee, A. Otto, and P. L. Pritchett, J. Geophys. Res. 106, 3715, DOI: 10.1029/1999JA900449 (2001).

${ }^{43}$ J. Birn and M. Hesse, J. Geophys. Res. 106, 3737, DOI: 10.1029/ 1999JA001001 (2001).

${ }^{44}$ M. Hesse, J. Birn, and M. Kuznetsova, J. Geophys. Res. 106, 3721, DOI: 10.1029/1999JA001002 (2001).

${ }^{45}$ J. D. Huba and L. I. Rudakov, Phys. Rev. Lett. 93, 175003 (2004).

${ }^{46}$ D. A. Knoll and L. Chacón, Phys. Rev. Lett. 96, 135001 (2006).

${ }^{47}$ D. A. Knoll and L. Chacón, Phys. Plasmas 13, 2307 (2006).

${ }^{48}$ Z. W. Ma and A. Bhattacharjee, J. Geophys. Res. 106, 3773, DOI: 10.1029/1999JA001004 (2001).

${ }^{49}$ A. Otto, J. Geophys. Res. 106, 3751, DOI: 10.1029/1999JA001005 (2001).

${ }^{50}$ P. L. Pritchett, J. Geophys. Res. 106, 3783, DOI: 10.1029/1999JA001006 (2001).

${ }^{51}$ M. A. Shay, J. F. Drake, B. N. Rogers, and R. E. Denton, J. Geophys. Res. 106, 3759, DOI: 10.1029/1999JA001007 (2001).

${ }^{52}$ L. Yin, D. Winske, S. P. Gary, and J. Birn, J. Geophys. Res. 106, 10761, DOI: 10.1029/2000JA000398 (2001).

${ }^{53}$ L. Chacón, D. A. Knoll, and J. M. Finn, Phys. Lett. A 308, 187 (2003).

${ }^{54}$ I. F. Shaikhislamov, J. Plasma Phys. 70, 599 (2004).

${ }^{55}$ R. B. Dahlburg, T. A. Zang, D. Montgomery, and M. Y. Hussaini, Proc. Natl. Acad. Sci. U.S.A. 80, 5798 (1983).

${ }^{56}$ N. Seehafer, E. Zienicke, and F. Feudel, Phys. Rev. E 54, 2863 (1996). 